\title{
Compreensão da Leitura: Análise do Funcionamento Diferencial dos Itens de um Teste de Cloze
}

\author{
Reading Comprehension: Differential Item Functioning Analysis of a Cloze Test
}

\author{
Katya Luciane Oliveira*, ${ }^{a}$, Acácia A. Aparecida Angeli dos Santos ${ }^{b}$, Evely Boruchovitch ${ }^{c}$ \\ \& Fabian Javier Marín Rueda ${ }^{b}$ \\ ${ }^{a}$ Universidade Estadual de Londrina, Londrina, Brasil, ${ }^{b}$ Universidade São Francisco, Itatiba, Brasil \\ $\&{ }^{c}$ Universidade Estadual de Campinas, Campinas, Brasil
}

\begin{abstract}
Resumo
Este estudo teve por objetivos investigar o ajuste de um Teste de Cloze ao modelo Rasch e avaliar a dificuldade na resposta ao item em razão do gênero das pessoas (DIF). Participaram da pesquisa 573 alunos das $5^{\mathrm{a}}$ a $8^{\mathrm{a}}$ séries do ensino fundamental de escolas públicas estaduais dos estados de São Paulo e Minas Gerais. O teste de Cloze foi aplicado de forma coletiva. A análise do instrumento evidenciou um bom ajuste ao modelo Rasch, bem como os itens foram respondidos conforme o padrão esperado, demonstrando um bom ajuste, também. Quanto ao DIF, apenas três itens indicaram diferenciar o gênero. Com base nos dados, identificou-se que houve equilíbrio nas respostas dadas pelos meninos e meninas. Palavras-chave: Teoria de resposta ao item, técnica de Cloze, compreensão em leitura.
\end{abstract}

\begin{abstract}
The objectives of the present study were to investigate the adaptation of a Cloze test to the Rasch Model as well as to evaluate the Differential Item Functioning (DIF) in relation to gender. The sample was composed by 573 students from $5^{\text {th }}$ to $8^{\text {th }}$ grades of public schools in the state of São Paulo. The cloze test was applied collectively. The analysis of the instrument revealed its adaptation to Rash Model and that the items were responded according to the expected pattern, showing good adjustment, as well. Regarding DIF, only three items were differentiated by gender. Based on the data, results indicated a balance in the answers given by boys and girls.

Keywords: Item response theory, Cloze technique, reading comprehension.
\end{abstract}

A leitura deve ser considerada uma das formas mais relevantes de aquisição do conhecimento, por se tratar de uma das habilidades exigidas nas atividades cotidianas. No contexto escolar, a leitura permite o desenvolvimento do pensamento crítico, instrumentalizando o aluno para o entendimento dos conteúdos escolares. Desse modo, é na escola e, especialmente, no ensino fundamental que ocorre um dos momentos mais importantes para o ensino da leitura (J. B. A. Oliveira, 2005; Salvia \& Ysseldyke, 1991; Souza, 2007; Zorzi, Serapompa, P. S. Oliveira, \& Faria, 2003).

A compreensão em leitura tem sido tema de muitas pesquisas na área. A ênfase na compreensão em leitura se deve ao fato de que a leitura não deve ser entendida somente como decodificação de signos lingüísticos, mas sim

\footnotetext{
* Endereço para correspondência: Departamento de Psicologia e Psicanálise, Universidade Estadual de Londrina, Rodovia Celso Garcia Cid, Pr. 445, km 3880, Londrina, PR, Brasil, 86055-900. E-mail: o 1 i v e i r a k a ty a@ig.com.br, acacia.santos@saofrancisco.edu.br,evely@unicamp.br e marinfabian@yahoo.com.br
}

uma habilidade que requer análise, síntese, criatividade e, sobretudo, compreensão das informações principais trazidas pelo texto (Cunha \& Santos, 2006; K. L. Oliveira \& Santos, 2005, 2006; K. L. Oliveira, Santos, \& Primi, 2003; Ruddell, Ruddell, \& Singer, 2001; Santos, 2004).

Embora os Parâmetros Curriculares Nacionais do Ensino de Língua Portuguesa (Ministério da Educação e Cultura [MEC], 1998) esclareçam que o aluno deve sair do ensino fundamental com um bom domínio da leitura, dados do Instituto Nacional de Estudos e Pesquisas Educacionais Anísio Teixeira ([INEP], 2003) revelam que os alunos das oitava séries do ensino fundamental apresentam uma habilidade de compreensão em leitura muito aquém do esperado para esse nível de escolaridade. Daí a necessidade de que investigações sobre o tema sejam realizadas continuamente.

Há grande dificuldade para se levantar o nível de compreensão em leitura de alunos das diferentes séries escolares, pois existe carência de instrumentos válidos para essa finalidade. Assim sendo, um recurso diagnóstico que vem ganhando destaque nas últimas décadas é o teste de Cloze, que pode ser caracterizado como um método efi- 
caz na mensuração da compreensão em leitura. O teste, criado por Taylor (1953), consiste em um método no qual utiliza um texto de aproximadamente 250 palavras e se omite todos os quintos vocábulos, no local da palavra omitida se coloca um traço de tamanho proporcional que deve ser preenchido pelo testando (McKenna \& Robinson, 1980).

De um modo geral, as pesquisas realizadas com o teste de Cloze no ensino fundamental (Gomes \& Boruchovitch, 2005; K. L. Oliveira, Boruchovitch, \& Santos, 2007, 2008; Santos, Rueda, \& Bartholomeu, 2006; Santos, Sampaio, Lukjanenko, Cunha, \& Zenorini, 2006) utilizam o escore bruto do teste, recorrendo à somatória das palavras preenchidas corretamente. Todavia, autores como Abraham e Chapelle (1992) discutem que os itens/lacunas que compõem o teste de Cloze apresentam diferentes níveis de dificuldades e, portanto, devem ser analisados dentro de suas especificidades. Para eles, a estruturação do texto preparado segundo a técnica de Cloze apresenta pelo menos dois níveis de dificuldade, quais sejam, micro nível (itens relativamente fáceis à compreensão) e macro nível (itens com um alto grau de dificuldade). Os determinantes da dificuldade implicadas em um item podem ser divididos de acordo com os fatores intrínsecos que se referem aos elementos que facilitam a execução da tarefa e estimulam a compreensão do item (no Cloze o tamanho do traço é proporcional ao tamanho da palavra omitida - pista contextual) e os fatores extrínsecos que abrangem os conhecimentos prévios que o aluno tem sobre o assunto.

Pode-se supor que no Cloze há um micro nível composto por itens/lacunas cujo preenchimento é mais fácil e também há um macro nível no qual exige do testando uma maior habilidade. Com base em evidências levantadas por Page (1975) e, mais recentemente, por Santos, Primi, Taxa e Vendramini (2002) constata-se que, no Teste de Cloze, itens heterogêneos não podem ter o mesmo peso. Nesse sentido, há concordância com Abraham e Chapelle (1992), que também apontam diferenças de dificuldades relativas a cada um dos itens, mostrando a necessidade de sua análise.

Recomendações advindas de entidades internacionais (American Educational Research Association [AERA], American Psychological Association [APA], \& National Council on Measurement in Education [NCME], 1999) destacam que o que se valida em um teste são os escores e não o teste em si, que é composto por diferentes itens que devem ser considerados dentro de cada grau específico de dificuldade de serem acertados. Com base nas considerações trazidas, o presente estudo propõe-se a utilizar a Teoria de Resposta ao Item - TRI para investigar funcionamento diferencial dos itens de um teste de Cloze.

Embretson e Reise (2000) consideram que a TRI permite levantar a dificuldade dos itens que compõe um determinado teste, levando à compreensão do porque alguns itens são mais fáceis e outros mais difíceis. Com isso é possível fazer conjecturas sobre a habilidade das pessoas, haja vista para o postulado da TRI de que as pessoas têm maior possibilidade de acertar um item fácil do que um difícil (Sisto, 2005, 2006a). Assim sendo, pessoas com maior habilidade são mais capazes de acertarem os itens e o acerto ou o erro devem ser produtos da pessoa e da posição do item, não podendo apresentar qualquer influência de alguma outra variável, tal como, etnia, gênero, grupo social, dentre outras.

Com a preocupação de aferir que o acerto de um dado item somente pode ser explicado em função da habilidade da pessoa ou pela dificuldade do item, Rasch (1960) criou um modelo de análise no qual é possível levantar algum viés na medida. As pesquisas realizadas pelo autor demonstraram que algumas pessoas, com equivalência na capacidade para responder um item, tinham diferentes possibilidades de obter êxito no acerto. Destarte, era possível que algum outro aspecto, viés, ou funcionamento diferencial dos itens - DIF, como proposto por Holland e Thayer (1988) pudesse interferir na probabilidade de acerto do item.

Sisto (2006a, 2006b) considera que um item uniforme garante a mesma probabilidade de acerto em dois grupos diferentes. Isso significa que o fato da pessoa fazer parte de algum grupo específico não pode ser fator determinante para ela acertar ou errar um dado item. Ainda, existem autores como Linacre (2002) que ressaltam que certa quantidade de DIF muitas vezes está presente, o que não necessariamente implicaria em um viés que levasse à retirada de itens ou reconfiguração do instrumento.

No caso do Cloze, a utilização do modelo de Rasch (1960) foi motivada, tendo em vista que as investigações de K. L. Oliveira (2008) e K. L. Oliveira et al. (2007) com alunos do ensino fundamental evidenciaram que havia diferença na pontuação do Cloze, considerando meninos e meninas. Os estudos citados fizeram uso do mesmo teste de Cloze utilizado na presente investigação e constataram que as meninas se saiam significativamente melhor no Cloze do que os meninos. Na ocasião questionou-se o fato do acerto ter ocorrido somente em razão da habilidade dos alunos e da dificuldade do item, ou se a probabilidade de acerto mudaria se o aluno fosse do gênero masculino ou feminino, o que se constituiria num viés na medida.

Com essa perspectiva, K. L. Oliveira, Santos, Boruchovitch e Rueda (2009) investigaram um teste de Cloze com 15 lacunas/itens elaborado para crianças das séries iniciais $\left(2^{\mathrm{a}}, 3^{\mathrm{a}}\right.$ e $\left.4^{\mathrm{a}}\right)$ do ensino fundamental. Observou-se que os itens mais fáceis foram os artigos e o mais difícil foi um substantivo. Os resultados indicaram que os itens foram respondidos de acordo com o padrão esperado, demonstrando bom ajuste ao modelo de Rasch. O texto 
elaborado não mostrou vieses nos itens, estudado por meio do DIF. Com base nisso, os autores ressaltaram não haver necessidade de serem criados critérios diferentes para meninos e meninas, haja vista que os itens não privilegiaram nenhum dos dois gêneros.

No que se refere ao teste de Cloze com 40 lacunas, pode-se fazer referência ao trabalho de Santos et al. (2002), que procurou analisar as propriedades psicométricas do teste de Cloze para a avaliação da compreensão em leitura em universitários. Os autores identificaram um ajuste dos itens do teste de acordo com os parâmetros da TRI, sendo destacado também a existência de dificuldades relativas à classe gramatical do vocábulo omitido. Dentro desse contexto, este estudo objetivou trabalhar com um teste de Cloze com o mesmo número de lacunas, porém com características adequadas a alunos dos últimos anos do Ensino Fundamental.

O trabalho se justifica, pois a técnica de Cloze, apesar de sua eficiência como meio diagnóstico para avaliar a compreensão em leitura, ainda está insuficientemente explorada no ensino fundamental (Santos et al., 2006; Zucoloto \& Sisto, 2002). Os estudos com o Cloze são em sua maioria de natureza descritiva de levantamento ou correlacional (Bitar, 1989; Gomes \& Boruchovitch, 2005; K. L. Oliveira, 2008; K. L. Oliveira et al., 2007, 2008; Santos et al., 2006). Poucas pesquisas nacionais e internacionais buscaram identificar as evidências de validade do teste de Cloze (Abraham \& Chapelle, 1992; Bensoussan, 1990; Chance, 1985, Hughes, 1994; Page, 1975; Santos, 2005; Santos et al., 2002). Assim, torna-se relevante investigar o teste de Cloze, de modo que as interpretações provenientes de sua utilização fiquem mais refinadas, denotando em um diagnóstico válido e confiável da compreensão em leitura.

Diante dos argumentos apresentados, são objetivos da presente pesquisa analisar a estrutura interna de um teste de Cloze para crianças de $5^{\mathrm{a}}$ a $8^{\mathrm{a}}$ séries do ensino fundamental por meio da TRI. Para tanto, se propõe a investigar o ajuste do Teste de Cloze ao modelo Rasch, assim como também verificar a existência de possíveis vieses nos itens do instrumento, por meio do funcionamento diferencial dos itens (DIF) em função do gênero das crianças.

\section{Método}

\section{Participantes}

Participaram 573 alunos de $5^{\mathrm{a}}(28,6 \%, n=164), 6^{\mathrm{a}}$ (28,4\%, $n=163), 7^{\text {a }}(20,8 \%, n=119)$ e $8^{\text {a }}(22,2 \%, n=127)$ séries do ensino fundamental, de escolas públicas esta- duais $(75,9 \% ; n=435)$ e privadas $(24,1 \% ; n=138)$ dos estados de São Paulo e Minas Gerais. A média de idade foi de 12 anos e 8 meses $(D P=1,2)$. Quanto ao gênero, $46,2 \%$ $(n=265)$ da amostra eram do gênero masculino e 53,8\% $(n=308)$ do feminino.

\section{Instrumentos}

Foi utilizado um texto preparado segundo a técnica de Cloze (Anexo A) de autoria de Santos (2003). O texto é destinado às crianças de $5^{\mathrm{a}}$ a $8^{\mathrm{a}}$ séries do ensino fundamental e é intitulado 'Coisas que acontecem'. O texto possuía 250 vocábulos e 40 omissões, sendo utilizada a forma de correção literal que aceitou como resposta correta o preenchimento exato da palavra que foi omitida Foi atribuído 1 para acerto e 0 para erro.

\section{Procedimento}

A presente pesquisa foi submetida e aprovada pelo Comitê de Ética em Pesquisa (CEP) da Faculdade de Ciências Médicas da Universidade Estadual de Campinas, sob o parecer 120/2005. Desse modo, todos os procedimentos éticos para a realização deste estudo foram seguidos, pois estão fundamentados nos dispositivos da Resolução 196/96 e complementares do Conselho Nacional de Saúde (1996).

As aplicações do teste Cloze foram realizadas coletivamente em sala de aula nas crianças cujos pais concordaram com a participação do filho, assinando o termo de consentimento livre e esclarecido. Assim, cada aplicação durou aproximadamente 30 minutos.

\section{Resultados e Discussão}

Os dados foram organizados em planilha e submetidos ao programa estatístico Winsteps para realizar a análise pelo modelo Rasch. Os resultados da análise do teste de Cloze e seu ajuste ao modelo Rasch podem ser vistos na Tabela 1 que apresenta dados da dificuldade dos itens, erros padrão, infits e outfits. Segundo Ziviani e Primi (2002), as estatísticas do infit e outfit são sumários de resíduos calculados para as pessoas e para os itens, baseados nas discrepâncias entre os valores preditos de acerto a partir do modelo de Rasch e o valor observado de fato. Acrescenta-se que o infit é resultante do valor ponderado e o oufit não. Portanto, o infit é um índice mais sensível quando os resíduos acontecem em itens, cuja dificuldade é mais próxima da habilidade dos sujeitos. Por sua vez, pelo fato do outfit não apresentar tal ponderação, é mais sensível a discrepâncias em itens, cuja dificuldade é mais distante da habilidade dos sujeitos. 
Psicologia: Reflexão e Crítica, 25(2), 221-229.

Tabela 1

Distribuição do Ajuste dos Itens ao Modelo Rasch

\begin{tabular}{|c|c|c|c|c|c|}
\hline Item & Classe & Dificuldade & Erro & Infit & Outfit \\
\hline resolveu & verbo & 4,56 & 0,27 & 1,00 & 0,96 \\
\hline tantas & pronome & 4,42 & 0,26 & 1,00 & 3,44 \\
\hline colega & substantivo & 4,42 & 0,26 & 1,02 & 1,89 \\
\hline fugirem & verbo & 2,96 & 0,14 & 0,96 & 0,80 \\
\hline dos & preposição & 2,58 & 0,13 & 1,01 & 1,10 \\
\hline parada & adjetivo & 2,46 & 0,12 & 0,92 & 0,84 \\
\hline sobrevivência & substantivo & 2,41 & 0,12 & 1,00 & 0,98 \\
\hline contou & verbo & 2,03 & 0,11 & 0,96 & 0,85 \\
\hline pedaço & substantivo & 1.68 & 0,10 & 1,14 & 1,19 \\
\hline deu & verbo & 1,43 & 0,10 & 0,98 & 0,96 \\
\hline assim & advérbio & 1,38 & 0,10 & 0,90 & 0,81 \\
\hline até & advérbio & 1,36 & 0,10 & 1,01 & 1,26 \\
\hline sempre & advérbio & 1,33 & 0,10 & 0,97 & 0,95 \\
\hline tentou & verbo & 1,28 & 0,10 & 1,01 & 0,95 \\
\hline mas & conjunção & 1,05 & 0,09 & 0,89 & 0,81 \\
\hline passarinho & substantivo & 1,01 & 0,09 & 1,13 & 2,17 \\
\hline mesmo & pronome & 1,01 & 0,09 & 1,03 & 1,12 \\
\hline o & pronome & 0,46 & 0,09 & 1,02 & 1,04 \\
\hline passarinho & substantivo & 0,02 & 0,10 & 1,16 & 1,30 \\
\hline ele & pronome & $-0,01$ & 0,10 & 1,07 & 1,07 \\
\hline aves & substantivo & $-0,12$ & 0,10 & 1,01 & 1,15 \\
\hline que & pronome & $-0,15$ & 0,10 & 0,89 & 0,83 \\
\hline passarinho & substantivo & $-0,27$ & 0,10 & 1,14 & 1,26 \\
\hline não & advérbio & $-0,30$ & 0,10 & 0,96 & 0,98 \\
\hline $\mathrm{de}$ & preposição & $-0,71$ & 0,10 & 1,03 & 1,09 \\
\hline de & preposição & $-0,93$ & 0,11 & 0,96 & 0,88 \\
\hline no & preposição & $-1,51$ & 0,13 & 0,90 & 0,84 \\
\hline na & preposição & $-1,55$ & 0,13 & 1,06 & 1,04 \\
\hline embora & advérbio & $-1,55$ & 0,13 & 0,90 & 0,82 \\
\hline se & conjunção & $-1,63$ & 0,13 & 0,85 & 0,73 \\
\hline do & preposição & $-2,40$ & 0,16 & 1,00 & 1,42 \\
\hline menos & advérbio & $-2,43$ & 0,16 & 0,90 & 0,73 \\
\hline água & substantivo & $-2,72$ & 0,18 & 0,90 & 0,67 \\
\hline e & conjunção & $-2,72$ & 0,18 & 0,95 & 1,11 \\
\hline na & preposição & $-2,83$ & 0,19 & 1,12 & 0,83 \\
\hline o & artigo & $-2,83$ & 0,19 & 0,88 & 0,71 \\
\hline $\mathrm{e}$ & conjunção & $-2,98$ & 0,20 & 0,97 & 1,18 \\
\hline $\mathrm{a}$ & artigo & $-3,14$ & 0,21 & 0,91 & 0,90 \\
\hline $\mathrm{e}$ & conjunção & $-3,38$ & 0,23 & 0,85 & 0,71 \\
\hline $\mathrm{e}$ & conjunção & $-3,67$ & 0,25 & 1,20 & 1,88 \\
\hline
\end{tabular}

Pode ser evidenciado na Tabela 1 que a dificuldade dos itens variou de 4,56 a $-3,67$, sendo esses valores correspondentes aos itens verbo 'resolveu' e conjunção 'e' respectivamente. Uma síntese desses resultados é apresentada na Tabela 2. Constata-se que a média
$(M=0,99 ; D P=0,19)$ do Infit quanto ao ajuste dos itens foi adequada, podendo-se interpretar que os itens foram respondidos de acordo com o padrão esperado, que é de 1,00 . 
Oliveira, K. L., Santos, A. A. A. A., Boruchovitch, E. \& Rueda, F. J. M. (2012). Compreensão da Leitura: Análise do Funcionamento Diferencial dos Itens de um Teste de Cloze.

Tabela 2

Parâmetros de Ajuste dos Itens

\begin{tabular}{lccc}
\hline Parâmetros & Infit & Outfit & Erro \\
\hline Média & 0,99 & 1,11 & 0,14 \\
$D P$ & 0,09 & 0,49 & 0,05 \\
Máximo & 1,20 & 3,44 & 0,27 \\
Mínimo & 0,85 & 0,67 & 0,09 \\
$1,3<>1,5$ & $0(0,0 \%)$ & $2(5,0 \%)$ & \\
$1,5<>2,0$ & $0(0,0 \%)$ & $2(5,0 \%)$ & \\
$<2,0$ & $0(0,0 \%)$ & $2(5,0 \%)$ & \\
\hline
\end{tabular}

Também pela Tabela 2 pode ser observado que os valores do infit ficaram entre 0,85 e 1,20 , enquadrando-se no intervalo de 0,70 a 1,30 , tido como um bom critério de ajuste. Ainda pode-se fazer referência a Linacre (2002), que afirma que o valor de 1,5 pode ser considerado como limite máximo para se aceitar um item. A média dessa dificuldade foi de $0,99(D P=0,09)$, sugerindo uma boa adequação ao modelo.

Em relação ao outfit, o valor médio foi de 1,11, $(D P=0,49)$ revelando também um ajuste adequado ao modelo. A variação observada foi de 0,67 a 3,44 , sendo que $5(12,5 \%)$ itens ficaram com valores discrepantes ao se considerar o parâmetro de ajuste de 1,50 apontado por Linacre (2002). O pronome 'tantas', os substantivos 'água', 'colega' e 'passarinho' e a conjunção 'e' foram itens que não se mostraram adequados ao modelo. Nessa perspectiva, observa-se que o erro no item 'água' foi cometido por pessoas cujas habilidades permitiriam seu acerto. Já no caso dos itens 'tantas', 'colega', 'passarinho' e 'e' estes foram acertados por pessoas cujas habilidades não sugeriram a possibilidade de acertá-los.

No que tange às pessoas, a maior parte dos valores de infit e outfit ficaram próximos da média adequada ao modelo, qual seja, 1,00. Os dados estão apresentados na Tabela 3.

Tabela 3

Parâmetros de Ajuste das Pessoas

\begin{tabular}{lccc}
\hline Parâmetros & Infit & Outfit & Erro \\
\hline Média & 1,00 & 1,06 & 0,45 \\
$D P$ & 0,27 & 1,06 & 0,03 \\
Máximo & 3,20 & 9,90 & 1,85 \\
Mínimo & 0,52 & 0,32 & 0,44 \\
$1,3<>1,5$ & $30(5,2 \%)$ & $24(4,1 \%)$ & \\
$1,5<>2,0$ & $21(3,6 \%)$ & $31(5,4 \%)$ & \\
$<2,0$ & $1(0,1 \%)$ & $39(6,8 \%)$ & \\
\hline
\end{tabular}

No infit, uma parte das pessoas $(3,8 \% ; n=22)$ apresentou escolhas desajustadas, tendo em vista que ficaram fora do parâmetro. As respostas dessas pessoas não corresponderam com o esperado no teste de Cloze, tendo em vista suas habilidades para completar a lacuna. Quanto ao outfit, a análise mostrou que $12,2 \% ;(n=70)$ das pessoas tiveram escolhas desajustadas. Assim sendo, os percentuais de desajuste podem ser considerados aceitáveis.

O agrupamento dos itens e das pessoas no teste pode ser visto na Tabela 4. A letra M, ao lado da divisória das informações das pessoas e dos itens, indica a localização da média. Em acréscimo, o ponto zero no Rasch é o ponto de dificuldade média, então valores negativos são mais fáceis e os positivos e altos são os mais difíceis.

Com os resultados da Tabela 4, evidenciou-se que a média das pessoas ficou próxima à média dos itens. Pode-se supor que a média de dificuldade dos itens ficou próxima da média da habilidade das pessoas, o que revela que o teste, de uma forma geral, se apresentou adequado para esse grupo de crianças. Os itens considerados mais difíceis foram o verbo 'resolveu', o pronome 'tantas' e o substantivo 'colega'. Ainda, considerando a afirmação de Linacre (2002) de que certa quantidade de DIF sempre está presente, optou-se por não retirar esses vocábulos.

Posteriormente, realizou-se a análise do funcionamento diferencial do item (DIF). Andriola (2001) destaca que há três aspectos importantes que justificam os estudos referentes ao DIF, a saber, a busca de causas que expliquem eventuais diferenças na resposta aos itens; a não utilização de itens em grupos com características diferentes, evitando-se o risco de prejudicar grupos em desvantagem; e controlar os fatores responsáveis pelo DIF para não construir outros itens com os mesmos problemas. Os resultados podem ser vistos na Tabela 5 que apresenta os acréscimos de DIF por gênero e as mudanças e valores de $t$ por item. 
Tabela 4

Mapa de Itens e Pessoas

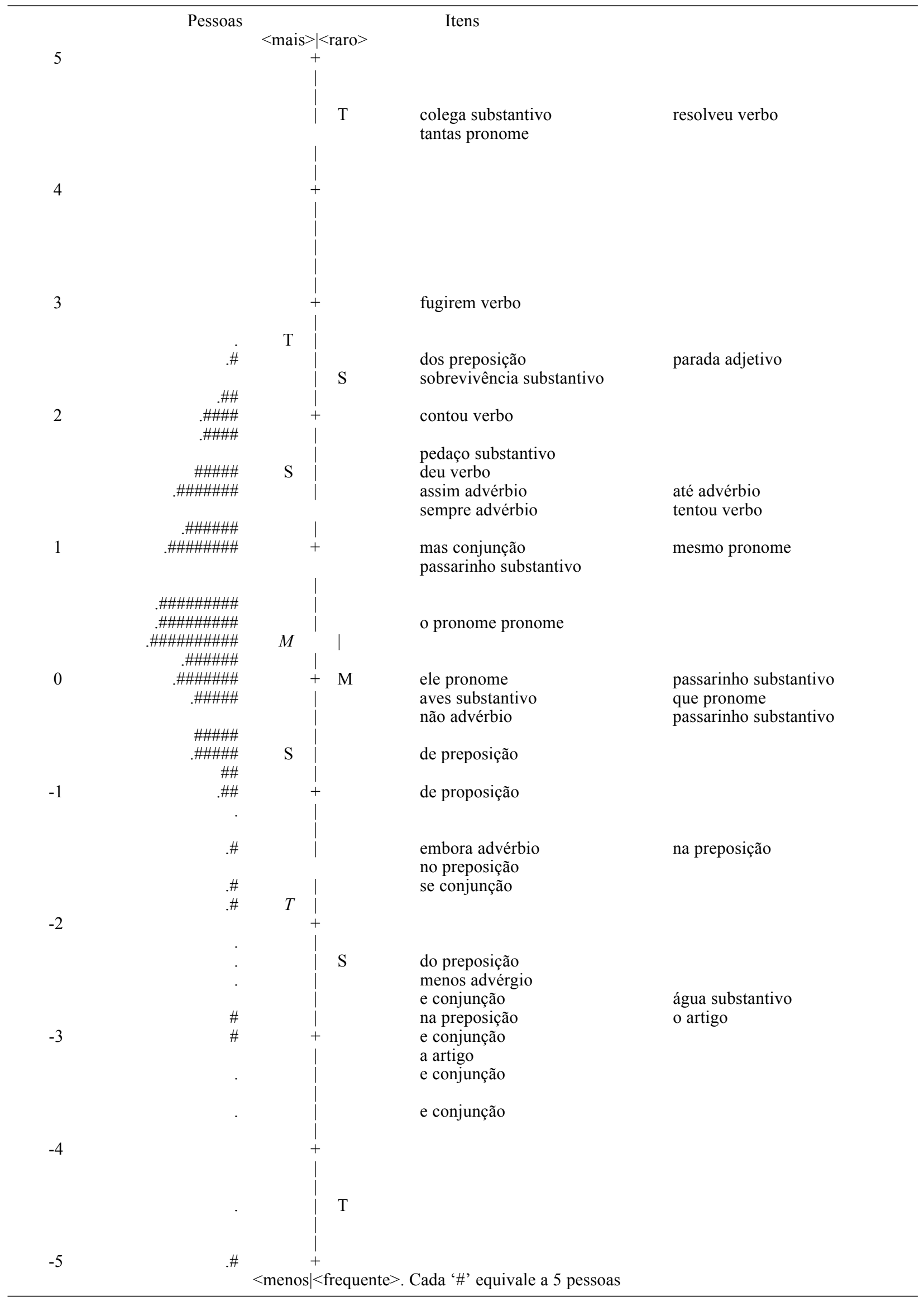


Oliveira, K. L., Santos, A. A. A. A., Boruchovitch, E. \& Rueda, F. J. M. (2012). Compreensão da Leitura: Análise do Funcionamento Diferencial dos Itens de um Teste de Cloze.

Tabela 5

Distribuição dos Acréscimos de DIF por Gênero de Valores de t por Item

\begin{tabular}{|c|c|c|c|c|c|c|c|}
\hline Gênero & $\begin{array}{c}\text { DIF } \\
\text { acrescido }\end{array}$ & Gênero & $\begin{array}{c}\text { DIF } \\
\text { acrescido }\end{array}$ & Mudança & $T$ & Item & Classe \\
\hline M & 0,03 & F & $-0,02$ & 0,05 & 0,24 & passarinho & Substantivo \\
\hline M & $-0,47$ & F & 0,50 & $-0,97$ & $-1,91$ & $\mathrm{e}$ & Conjunção \\
\hline M & $-0,01$ & $\mathrm{~F}$ & 0,01 & $-0,02$ & $-0,07$ & parada & Adjetivo \\
\hline M & 0,01 & F & $-0,01$ & 0,01 & 0,06 & até & Advérbio \\
\hline M & $-0,11$ & F & 0,12 & $-0,23$ & $-0,93$ & na & Preposição \\
\hline M & 0,14 & $\mathrm{~F}$ & $-0,21$ & 0,35 & 1,01 & menos & Advérbio \\
\hline M & 0,05 & F & $-0,03$ & 0,08 & 0,43 & mesmo & Pronome \\
\hline M & $-0,54$ & F & 0,52 & $-1,06$ & $-2,78$ & na & Preposição \\
\hline M & 0,07 & F & $-0,07$ & 0,14 & 0,69 & de & Preposição \\
\hline M & 0,18 & F & $-0,24$ & 0,42 & 1,56 & se & Conjunção \\
\hline M & 0,49 & $\mathrm{~F}$ & $-0,19$ & 0,68 & 1,02 & resolveu & Verbo \\
\hline M & $-0,19$ & F & 0,16 & $-0,34$ & $-1,80$ & passarinho & Substantivo \\
\hline M & $-0,23$ & $\mathrm{~F}$ & 0,25 & $-0,48$ & $-1,48$ & do & Preposição \\
\hline M & 0,09 & F & $-0,06$ & 0,15 & 0,69 & pedaço & Substantivo \\
\hline M & $-0,21$ & F & 0,21 & $-0,42$ & $-1,69$ & no & Preposição \\
\hline M & $-0,22$ & F & 0,13 & $-0,41$ & $-2,11$ & passarinho & Substantivo \\
\hline M & 0,11 & F & $-0,07$ & 0,18 & 0,90 & sempre & Advérbio \\
\hline M & 0,25 & F & $-0,51$ & 0,76 & 1,47 & e & Conjunção \\
\hline M & 0,39 & F & $-0,83$ & 1,22 & 2,77 & água & Substantivo \\
\hline M & $-0,09$ & F & 0,12 & $-0,21$ & $-0,56$ & 0 & Artigo \\
\hline M & 0,04 & F & $-0,07$ & 0,11 & 0,29 & $\mathrm{e}$ & Conjunção \\
\hline M & $-0,04$ & F & 0,03 & $-0,07$ & $-0,39$ & ele & Pronome \\
\hline M & $-0,01$ & F & 0,01 & $-0,02$ & $-0,05$ & $\mathrm{e}$ & Conjunção \\
\hline M & 0,15 & $\mathrm{~F}$ & $-0,27$ & 0,42 & 0,95 & $\mathrm{a}$ & Artigo \\
\hline M & 0,21 & $\mathrm{~F}$ & $-0,19$ & 0,40 & 2,04 & aves & Substantivo \\
\hline M & $-0,05$ & $\mathrm{~F}$ & 0,04 & $-0,09$ & $-0,47$ & tentou & Verbo \\
\hline M & 0,04 & $\mathrm{~F}$ & $-0,03$ & 0,08 & 0,41 & mas & Conjunção \\
\hline M & 0,26 & $\mathrm{~F}$ & $-0,36$ & 0,63 & 2,36 & embora & Advérbio \\
\hline M & 0,09 & $\mathrm{~F}$ & $-0,07$ & 0,17 & 0,88 & o & Pronome \\
\hline M & 0,00 & $\mathrm{~F}$ & 0,00 & 0,00 & 0,01 & deu & Verbo \\
\hline M & $-0,09$ & $\mathrm{~F}$ & 0,05 & $-0,14$ & $-0,27$ & tantas & Pronome \\
\hline M & $-0,09$ & $\mathrm{~F}$ & 0,08 & $-0,17$ & $-0,85$ & não & Advérbio \\
\hline M & 0,00 & $\mathrm{~F}$ & 0,00 & 0,00 & 0,01 & contou & Verbo \\
\hline M & 0,33 & $\mathrm{~F}$ & $-0,14$ & 0,47 & 0,80 & colega & Substantivo \\
\hline M & $-0,12$ & $\mathrm{~F}$ & 0,12 & $-0,25$ & $-1,13$ & de & Preposição \\
\hline M & 0,23 & F & $-0,22$ & 0,46 & 2,32 & que & Pronome \\
\hline M & $-0,10$ & $\mathrm{~F}$ & 0,06 & $-0,16$ & $-0,60$ & dos & Preposição \\
\hline M & 0,11 & $\mathrm{~F}$ & $-0,06$ & 0,17 & 0,56 & fugirem & Verbo \\
\hline M & $-0,10$ & $\mathrm{~F}$ & 0,07 & $-0,18$ & $-0,90$ & assim & Advérbio \\
\hline M & $-0,41$ & $\mathrm{~F}$ & 0,31 & $-0,73$ & $-3,00$ & sobrevivência & Substantivo \\
\hline
\end{tabular}

Dos 40 itens avaliados, apenas três revelaram que são diferenciados em razão do gênero. Assim, detectou-se que a preposição 'na' e o substantivo 'sobrevivência', foram mais fáceis para as meninas do que para os meninos. Entretanto, o substantivo 'água' mostrou-se mais fácil para os meninos do que para as meninas. Como apenas esses itens discriminaram os gêneros, concluiu-se que houve equilíbrio nos vieses ocorridos. Assim sendo, conside- rou-se que não há necessidade de substituir ou excluir os itens e que não seria necessário um critério de correção diferente para meninos e meninas.

Esses dados estão coerentes com os resultados obtidos por K. L. Oliveira et al. (2009) que estudaram um teste destinado a crianças das $2^{\mathrm{a}}$ a $4^{\mathrm{a}}$ séries do ensino fundamental. Os autores também evidenciaram que os itens do Cloze se apresentaram com um bom ajuste e não discri- 
minavam os gêneros. Embora o texto utilizado por K. L. Oliveira et al. (2009) tenha apresentado menos omissões (15) do que a presente pesquisa (40), os resultados foram semelhantes. Estudos como estes confirmam a validade do teste de Cloze estudado como meio confiável de medida, especialmente, quando se considera que os itens não mostraram vieses (Rueda, 2007; Sisto, 2006a).

\section{Considerações Finais}

A presente pesquisa, tendo por base resultado de estudos anteriores, hipotetizou que poderia haver diferença nos itens do teste de Cloze, sendo que alguma diferença poderia ser mais comum em um ou outro gênero. Portanto, levantou-se o funcionamento diferencial dos itens (DIF) que compunham o teste de Cloze, utilizando o modelo de Rasch (1960) que permite investigar a probabilidade de acerto aos itens, por pessoas com determinada habilidade, tendo em vista suas dificuldades.

Pode-se considerar que estudos que se propõem a analisar os itens, recorrendo a TRI como meio de análise, permitem aferir evidência de validade de construto ao instrumento analisado, pois evidenciam o quanto cada item estaria contribuindo para mensurar o construto. Um outro aspecto a ser mencionado é a análise do DIF, que possibilitou concluir que a medida de compreensão investigada na presente pesquisa não apresentou vieses de gênero, não havendo necessidade de modificá-la.

Esses dados reforçam a confiabilidade do uso da técnica de Cloze na mensuração da compreensão em leitura, ampliando as considerações acerca de sua eficácia. Todavia, não se descarta a necessidade de investigações futuras que visem aferir novas evidências de validade para o teste de Cloze.

\section{Referências}

Abraham, R. G., \& Chapelle, C. A. (1992). The meaning of Cloze test scores: An item difficulty perspective. The Modern Language Journal, 76, 468-479.

American Educational Research Association, American Psychological Association, \& National Council on Measurement in Education. (1999). Standards for educational and psychological testing. Washington, DC: American Psychological Association.

Andriola, W. B. (2001). Descrição dos principais métodos para detector o funcionamento diferencial dos itens (DIF). Psicologia: Reflexão e Crítica, 14(3), 643-652.

Bensoussan, M. (1990). Redundancy and the cohesion Cloze. Journal of Research in Reading, 13(1), 18-37.

Bitar, M. L. (1989). Eficiência dos instrumentos de avaliação em leitura. (Dissertação de Mestrado não-publicada). Pontifícia Universidade Católica de São Paulo, SP.

Chance, L. (1985). Use Cloze encounters of the readability kind for secondary school students. Journal of Reading, 29 , 690-693.

Conselho Nacional de Saúde. (1996). Resolução 196/96. Diretrizes e normas regulamentadoras de pesquisa. Retrieved October 19, 2005, from http://www.conselho.saude.gov.br
Cunha, N. B., \& Santos, A. A. A. (2006). Relação entre a compreensão da leitura e a produção escrita em universitários. Psicologia: Reflexão e Crítica, 19(2), 237-245.

Embretson, S. E., \& Reise, S. P. (2000). Item response theory for psychologists. Mahwah, NJ: Lawrence Erblbaum.

Gomes, M. A. M., \& Boruchovitch, E. (2005). Desempenho no jogo, estratégias de aprendizagem e compreensão na leitura. Psicologia: Teoria e Pesquisa, 21(3), 319-326.

Holland, P. W., \& Thayer, D. T (1988). Differential item performance and the Mantel-Haenszel procedure. In H. Wainer \& H. I. Braum (Eds.), Test validity (pp. 332-345). Hillsdale, NJ: Lawrence Erlbaum.

Hughes, A. (1994). Testing for language teachers. Cambridge, MA: Cambridge University Press.

Instituto Nacional de Estudos e Pesquisas Educacionais Anísio Teixeira. (2003). Qualidade da educação: Uma nova leitura do desempenho dos estudantes de $8^{a}$. série do ensino fundamental. Retrieved November 12, 2007, from http:// www.inep.gov.br/download/saeb/2003/boletim_8serie.pdf

Linacre, J. M. (2002). What do infit na outfit, mean-square and standardized mean? Rasch Measurement Transactions, $16(2), 887$.

McKenna, M. C., \& Robinson, S. D. (1980). An introduction to the Cloze procedure: An annotated bibliography. Newmark, DE: International Reading Association.

Ministério da Educação e Cultura. (1998). Parâmetros curriculares nacionais: Língua portuguesa: Ensino de quinta a oitava séries. Retrieved November 12, 2007, from http:/ /portal.mec.gov.br/seb/arquivos

Oliveira, J. B. A. (2005). Avaliação em educação. Avaliação: Ensaio e Políticas Públicas em Educação, 13(48), 375-382.

Oliveira, K. L. (2008). Escala de estratégias de aprendizagem para o ensino fundamental: Análise de suas propriedades psicométricas. (Tese de Doutorado não-publicada). Faculdade de Educação, Universidade Estadual de Campinas, SP.

Oliveira, K. L., Boruchovitch, E., \& Santos, A. A. A. (2007). Compreensão de leitura em alunos de sétima e oitava séries do ensino fundamental. Psicologia Escolar e Educacional, 11(2), 41-49.

Oliveira, K. L., Boruchovitch, E., \& Santos, A. A. A. (2008). Leitura e desempenho escolar em português e matemática no ensino fundamental. Paidéia, 18(41), 531-540.

Oliveira, K. L., \& Santos, A. A. A. (2005). Compreensão em leitura e avaliação da aprendizagem em universitários. Psicologia: Reflexão e Crítica, 18(1), 118-124.

Oliveira, K. L., \& Santos, A. A. A. (2006). Compreensão de textos e desempenho acadêmico Psic-Vetor, 7(1), 19-27.

Oliveira, K. L., Santos, A. A. A., Boruchovitch, E., \& Rueda, F. J. M. (2009). O teste de Cloze no ensino fundamental: Funcionamento diferencial do item. Manuscrito não-publicado.

Oliveira, K. L., Santos, A. A. A., \& Primi, R. (2003). Estudo das relações entre compreensão em leitura e desempenho acadêmico na universidade. Interação em Psicologia, 7(1), 19-25.

Page, W. D. (1975). The post oral reading Cloze test: New link between oral reading and comprehension. Journal of Reading Behavior, 7(4), 385-389.

Rasch, G. (1960). Probabilistic models for some intelligence and attainment tests. Copenhagen, Denmark: Danmarks Paedogogiske Institut.

Ruddell, R. B., Ruddell, M. R., \& Singer, H. (2001). Theoretical models and process of reading. Newark, DE: International Reading Association. 
Oliveira, K. L., Santos, A. A. A. A., Boruchovitch, E. \& Rueda, F. J. M. (2012). Compreensão da Leitura: Análise do Funcionamento Diferencial dos Itens de um Teste de Cloze.

Rueda, F. J. M. (2007). O funcionamento diferencial do item no Teste Pictórico de Memória. Avaliação Psicológica, 6(2), 229-237.

Salvia, J., \& Ysseldyke, J. (1991). Avaliação em educação especial e corretiva. São Paulo, SP: Manole.

Santos, A. A. A. (2003). Coisas que acontecem. Texto preparado segundo a técnica de Cloze para crianças de $5^{a}$ a $8^{a}$ séries do ensino fundamental. Manuscrito não-publicado, Programa de Pós-graduação Stricto Sensu, Universidade São Francisco, Itatiba, SP.

Santos, A. A. A. (2004). O Cloze como técnica de diagnóstico e remediação da compreensão em leitura. Interação em Psicologia, 8(2), 217-226.

Santos, A. A. A. (2005). O Teste de Cloze como instrumento de diagnóstico e de desenvolvimento da compreensão em leitu$r a$. Relatório técnico não-publicado, Programa de Pós-Graduação Stricto Sensu em Psicologia, Universidade São Francisco, Itatiba, SP.

Santos, A. A. A., Primi, R., Taxa, F., \& Vendramini, C. M. M. (2002). O teste de Cloze na avaliação da compreensão em leitura. Psicologia: Reflexão e Crítica, 15(3), 549-560.

Santos, A. A. A., Rueda, F. J. M., \& Bartolomeu, D. (2006). Avaliação dos aspectos afetivos envolvidos nas dificuldades de aprendizagem. In F. F. Sisto \& S. C. Martinelli (Eds.), Afetividade e dificuldades de aprendizagem (pp. 93-110). São Paulo, SP: Vetor.

Santos, A. A. A., Sampaio, I. S., Lukjanenko, M. F. S., Cunha, N. B., \& Zenorini, R. P. C. (2006). Avaliação de dificuldades em compreensão de leitura e escrita. In C. Machado, L. S. Almeida, M. Gonçalves, \& V. Ramalho (Eds.), XI Conferência Internacional de Avaliação Psicológica: Formas e Contextos (pp. 29-36). Braga, Portugal: Psiquilíbrios.
Sisto, F. F. (2005). Um estudo sobre a dimensionalidade do teste do Desenho da Figura Humana. Interação em Psicologia, 9(1), 11-19

Sisto, F. F. (2006a). Estudo do funcionamento diferencial dos itens para avaliar o reconhecimento de palavras. Avaliação Psicológica, 5(1), 1-10.

Sisto, F. F. (2006b). O funcionamento diferencial dos itens. Psico-USF, 11(1), 35-43.

Souza, L. (2007). A importância da leitura para a formação de uma sociedade consciente. Retrieved November 15, 2007, from http://dici.ibict.br/archive/00001095/01/ aimportanciadaleitura.pdf

Taylor, W. L. (1953). Cloze procedures: A new tool for measuring readability. Journalism Quarterly, 30, 415-433.

Ziviani, C., \& Primi, R. (2002). Teoria da resposta ao item e o modelo Rasch de mensuração: Uma análise do provão de psicologia. In R. Primi (Ed.), Temas em Avaliação Psicológica. Campinas, SP: Instituto Brasileiro de Avaliação Psicológica.

Zorzi, J. L., Serapompa, M. T., Oliveira, P. S., \& Faria, A. T. (2003). Aspectos da formação de leitores nas quatro séries iniciais do primeiro grau. Revista Psicopedagogia, 20(62), 189-201.

Zucoloto, K. A., \& Sisto, F. F. (2002). Dificuldades de aprendizagem na escrita e compreensão de leitura. Interação em Psicologia, 6(2), 157-166.

\section{Anexo A}

\section{Coisas da natureza}

Diogo costuma ficar lendo na sala de sua casa. Certa vez, próximo da meia-noite, levou um susto daqueles quando ouviu um barulho de algo batendo no vidro da janela. Quando olhou viu um

ficou observando a ave noite seguinte, mais ou no beiral da janela, à mesma hora, $\mathrm{o}$ que ele foi embora. Diogo parou de ler

Recebido: $10 / 08 / 2009$

$I^{a}$ revisão: $25 / 02 / 2010$ Aceite final: 15/02/2011

ficou bicando leve o vidro, como estivesse batendo para entrar

Diogo ___ ganhar a confiança do

um de fruta e água __ beiral da janela. $\mathrm{O}$
janela e pedacinhos da fruta _ bebeu um pouco da

Toda noite Diogo repetia _ ritual. Colocava alguma fruta água para o passarinho. Na terceira noite, antes __ passarinho chegar colocou
chegou na hora de $\quad$ Comeu Na terceira noite, antes __ passarinho chegar colocou
chegou na hora de . Comeu ficava por ali movendo cabeça como fazem as

Depois de alguns dias Diogo se aproximar do passarinho, de comer e beber ele voou e foi chegava comia e bebia irritado, achando-_ um ingrato, pois ele seu amigo.

essa história a um , cujo pai era criador aves. Ouviu como explicação faz parte da natureza passarinhos serem ariscos e dos seres humanos. É que conseguem garantir a da espécie!

\footnotetext{
${ }^{1}$ Texto de Cloze especialmente criado por Acácia A. Angeli dos Santos para avaliação da compreensão em leitura.
} 